\title{
NOTES
}

\section{Meso and Racemo Sequence Distribution of Polyacrylonitrile Chains Polymerized by Radical Polymerization with Redox Catalyst and $\gamma$-Ray Irradiation on Acrylonitrile-Urea Canal Complex}

\author{
Hitoshi YamaZaKI, Yukio MiYAZAKI, and Kenji KAMIDE* \\ Fundamental Research Laboratory of Fibers and Fiber-Forming Polymers, \\ Asahi Chemical Industry Company, Ltd., \\ Hacchonawate, 11-7, Takatsuki, Osaka 569, Japan
}

(Received June 30, 1986)

\author{
KEY WORDS Polyacrylonitrile / Pentad Tacticity / ${ }^{13} \mathrm{C}$ NMR Spectra \\ Meso Sequence Distribution / Racemo Sequence Distribution \\ Acrylonitrile-Urea Canal Complex / Gamma Ray / Redox Catalyst /
}

Recently Kamide et al. ${ }^{1}$ determined the pentad tacticity of polyacrylonitrile (PAN), polymerized using redox catalyst (hereafter referred to as R-PAN), by analyzing ${ }^{13} \mathrm{C}$ NMR spectrum measured in deuterated dimethylformamide $\left(\mathrm{DMF}-d_{7}\right)$. On the basis of the method established there, they ${ }^{2,3}$ determined the pentad tacticity of PAN, polymerized by $\gamma$-ray irradiation of acrylonitrileurea canal complex at $-78^{\circ} \mathrm{C}(\gamma$-PAN $)$. They disclosed that in the radical polymerization process with redox catalyst the propagation of meso- and racemo-sequences obeys Bernoulli statistics with the probability of meso dyad to the polymer chain end $P_{m}=0.519^{1}$ and the propagation process of $\gamma$-PAN chain is evidently controlled by the 1-st order Markov statistics and under the typical polymerization conditions the probability of linking meso sequence to racemo sequence $P(\mathrm{~m} / \mathrm{r})$ and the similar probability of racemo sequence to meso sequence $P(\mathrm{r} / \mathrm{m})$ were found to be 0.518 and 0.197 , respectively. $^{3}$
This note intends as an extension of the previous works ${ }^{1-3}$ to visualize the meso and racemo sequence distribution by generating in an electronic computer a number of PAN molecules with the meso-, racemo-sequences, giving the experimental values of $P_{\mathrm{m}}$ for RPAN and those of $P(\mathrm{~m} / \mathrm{r})$ and $P(\mathrm{r} / \mathrm{m})$ for $\gamma-$ PAN.

\section{EXPERIMENTAL}

The pentad tacticity data on an R-PAN whole polymer (sample code R-PAN-1, the weight-average molecular weight $\bar{M}_{w}=16.8$ $\times 10^{4}$ ) and a $\gamma$-PAN whole polymer (sample code $\gamma$-PAN-1, $\left.\quad \bar{M}_{w}=17.0 \times 10^{4}\right)$, prepared and evaluated in the previous papers ${ }^{1-3}$ were employed. Here, only three $\gamma$-PAN fractions $\left(\bar{M}_{w}=9.8 \sim 34.8 \times 10^{4}\right)$ isolated from the whole polymer were analyzed, revealing almost the same pentad tacticity (see Table I of ref 3) and we regarded the pentad tacticity averaged over these fractions as that for the unfractionated sample $(\gamma$-PAN-1).

\footnotetext{
* To whom all correspondences should be addressed.
} 
Table I. The pentad fractions and $P(\mathrm{~m} / \mathrm{r})$ and $P(\mathrm{r} / \mathrm{m})$ for polyacrylonitrile samples used

\begin{tabular}{|c|c|c|c|}
\hline \multirow{2}{*}{$\begin{array}{l}\text { Pentad } \\
\text { sequence }\end{array}$} & \multicolumn{3}{|c|}{ Pentad fraction } \\
\hline & $\left(\bar{M}_{w}^{\gamma-\mathrm{PAN}-1^{\mathrm{a}}}=17.0 \times 10^{4}\right)$ & $\begin{array}{c}\gamma \text {-PAN-2 } \\
\left(\bar{M}_{w}=18.4 \times 10^{4}\right)\end{array}$ & $\begin{array}{c}\text { R-PAN-1 } \\
\left(\bar{M}_{w}=16.8 \times 10^{4}\right)\end{array}$ \\
\hline $\mathrm{mmmm}$ & 0.374 & 0.586 & 0.077 \\
\hline $\mathrm{mmmr}$ & 0.115 & 0.085 & 0.125 \\
\hline rmmr & 0.040 & 0.031 & 0.068 \\
\hline $\mathrm{mmrm}$ & 0.118 & 0.079 & 0.125 \\
\hline mmrr & 0.073 & 0.056 & 0.097 \\
\hline $\mathrm{rmrm}$ & 0.087 & 0.062 & 0.149 \\
\hline $\mathrm{rmrr}$ & 0.055 & 0.028 & 0.129 \\
\hline mrrm & 0.029 & 0.008 & 0.055 \\
\hline mrrr & 0.051 & 0.019 & 0.112 \\
\hline rrrr & 0.057 & 0.046 & 0.065 \\
\hline$P(\mathrm{~m} / \mathrm{r})$ & 0.518 & 0.469 & $0.519\left(\equiv P_{\mathrm{m}}\right)$ \\
\hline$P(\mathrm{r} / \mathrm{m})$ & 0.197 & 0.100 & $0.481\left(\equiv 1-P_{\mathrm{m}}\right)$ \\
\hline
\end{tabular}

a Averaged tacticity for three fractions in Table I of ref 3.

b Table II of ref 2 .

Another $\gamma$-PAN sample was also prepared by $\gamma$-ray irradiation on urea-canal complex of acrylonitrile under somewhat different conditions $^{4}$ (The acrylonitrile-urea canal complex $(1 / 1.5, \mathrm{~mol} / \mathrm{mol})$ was irradiated with $\gamma$-ray from cobalt 60 at $-78^{\circ} \mathrm{C}$ at a dose rate of $0.6 \times 10^{5} \mathrm{R} \mathrm{h}^{-1}$ for $3 \mathrm{~h}$, the irradiation dose was $0.18 \mathrm{MR}$ ), and referred to as sample code $\gamma$-PAN-2. The unfractionated sample had $\bar{M}_{w}=18.4 \times 10^{4}$ and used for the further study. The pentad tacticity of $\gamma$-PAN-2 was evaluated, according to the method proposed by Kamide et al., ${ }^{1}$ from ${ }^{13} \mathrm{C}$ NMR spectrum in deuterated dimethyl sulfoxide (DMSO- $d_{6}$ ) solution at $80^{\circ} \mathrm{C}$. The results are shown in Table I, in which the data are also compiled for comparison on $\gamma$-PAN-1 and R-PAN samples. ${ }^{2,3}$

The content of mmmm (m: meso configuration) of sample code $\gamma-\mathrm{PAN}-2$ is about 1.5 times larger than that of $\gamma-\mathrm{PAN}-1$ and in fact 7.6 times larger than R-PAN. From the contents of various pentad sequences determined in this way, $P(\mathrm{~m} / \mathrm{r})$ and $P(\mathrm{r} / \mathrm{m})$ for sample code $\gamma$-PAN-2 were calculated by Kamide et al.'s procedure $^{2}$ to be 0.469 and 0.100 , respective- ly. These values are compared with 0.518 and 0.197 for sample $\gamma$-PAN-1.

Using these $P(\mathrm{~m} / \mathrm{r})$ and $P(\mathrm{r} / \mathrm{m})$ (note $P_{m}=$ $P(\mathrm{~m} / \mathrm{r})$ for R-PAN-1) values for those three sample codes, PAN molecules were artificially generated by Monte Carlo method in an electronic computer. The calculations were performed 1000 times, (in other words, a thousand molecules were generated for each sample code) and the degree of polymerization $\mathrm{N}$ was adjusted to give 4001 (i.e., the molecular weight $M=21.2 \times 10^{4}$ ).

\section{RESULTS AND DISCUSSION}

For quantitative understanding of the characteristics of meso- and racemo-sequences in a given chain of PAN samples, prepared by different methods, we intended to evaluate the number of meso sequences with $p$ meso dyad $\left(\mathrm{m}_{1} \mathrm{~m}_{2} \mathrm{~m}_{3} \cdots \mathrm{m}_{p}\right) F_{\mathrm{m}}(p)$ and the number of racemo sequences with $q$ racemo dyad $\left(\mathrm{r}_{1} \mathrm{r}_{2} \mathrm{r}_{3} \cdots \mathrm{r}_{q}\right) F_{r}(q)$, both for a given molecules:

Here, the most probable $F_{\mathrm{m}}(p)$ and $F_{\mathrm{r}}(q)$, $\overline{F_{\mathrm{m}}(p)}$ and $\overline{F_{\mathrm{r}}(q)}$ are defined by 


$$
\begin{gathered}
\overline{F_{\mathrm{m}}(p)}=\bar{B}_{\mathrm{m}} P_{\mathrm{A}} P_{\mathrm{B}}^{p-1} \\
\overline{F_{\mathrm{r}}(q)}=\bar{B}_{\mathrm{r}} P_{\mathrm{C}} P_{\mathrm{D}}^{q-1}
\end{gathered}
$$

with

$$
\begin{gathered}
\bar{B}_{m}=\sum_{p=1}^{N-1} \overline{F_{m}(p)} /\left(1-P_{\mathrm{B}}{ }^{N-1}\right) \\
\bar{B}_{r}=\sum_{q=1}^{N-1} \overline{F_{r}(q)} /\left(1-P_{\mathrm{D}}{ }^{N-1}\right)
\end{gathered}
$$

where $B_{\mathrm{m}}$ and $B_{\mathrm{r}}$ are the total number of meso- and racemo-sequence blocks in the molecule $\left(B_{\mathrm{m}}=B_{\mathrm{r}}\right.$ or $\left.B_{\mathrm{r}} \pm 1\right)\left(\bar{B}_{\mathrm{m}}\right.$ and $\bar{B}_{\mathrm{r}}$ are the average values), $P_{\mathrm{A}}=P(\mathrm{r} / \mathrm{m}), P_{\mathrm{B}}=1-P$ $(\mathrm{r} / \mathrm{m}), \quad P_{\mathrm{C}}=P(\mathrm{~m} / \mathrm{r})$ and $P_{\mathrm{D}}=1-P(\mathrm{~m} / \mathrm{r})$ for $\gamma$-PAN and $P_{\mathrm{A}}=P_{\mathrm{D}}=1-P_{\mathrm{m}}, \quad P_{\mathrm{B}}=P_{\mathrm{C}}=P_{\mathrm{m}}$ for R-PAN.

The following relation holds between

$$
\begin{gathered}
\sum_{p=1}^{N-1} \overline{F_{m}(p)} \text { and } \sum_{q=1}^{N-1} \overline{F_{r}(q)} ; \\
\sum_{p=1}^{N-1} \overline{F_{m}(p)} \cdot p+\sum_{q=1}^{N-1} \overline{F_{r}(q)} \cdot q=N-1
\end{gathered}
$$

Then, for large $N$ we can consider $B_{\mathrm{m}}=B_{\mathrm{r}}$, then, substitution of eq 1,2 into eq 5 gives

$$
\bar{B}_{m}=\bar{B}_{r}=\frac{N-1}{\sum_{p=1}^{N-1}\left(P_{\mathrm{A}} P_{\mathrm{B}}^{p-1} \cdot p\right)+\sum_{q=1}^{N-1}\left(P_{\mathrm{C}} P_{\mathrm{D}}^{q-1} \cdot q\right)}
$$

Using the experimental $P_{\mathrm{A}}, P_{\mathrm{B}}, P_{\mathrm{C}}$, and $P_{\mathrm{D}}$ data determined by NMR analysis $\bar{B}_{\mathrm{m}}$ (and $\bar{B}_{\mathrm{r}}$ ) can be calculated from eq 6 for the PAN polymer with $N$.

In the previous paper ${ }^{3}$ averaged values of $B_{\mathrm{m}}$ and $B_{\mathrm{r}}$ over 100 molecules with $N=4001$ were calculated by Monte Carlo method for R- and $\gamma$-PAN-1 samples. These values were in an excellent agreement with $\bar{B}_{\mathrm{m}}$ and $\bar{B}_{\mathrm{r}}$ calculated from eq 6 within relative error of $\pm 1 \%$. Of course, $\bar{B}_{\mathrm{m}}$ and $\bar{B}_{\mathrm{r}}$ evaluated here from 1000 molecules by Monte Carlo method also agree with those from eq 6 .

$$
\overline{F_{\mathrm{m}}(p)} \text { and } \overline{F_{\mathrm{r}}(q)} \text { can then be readily calcu- }
$$

lated from eq 1 and 2, respectively. The results are shown in Figure 1 as full line.

Alternatively, $F_{\mathrm{m}}(p)$ and $F_{\mathrm{r}}(q)$ can be directly estimated for each molecule by Monte Carlo method and accordingly $\overline{F_{\mathrm{m}}(p)}$ and $\overline{F_{\mathrm{r}}(q)}$ coincide with $F_{\mathrm{m}}(p)$ and $F_{\mathrm{r}}(q)$, a veraged over a large number of molecules:

$$
\overline{F_{m}(p)}=\frac{\sum_{i=1}^{1000} F_{m}^{i}(p)}{1000} \text { and } \overline{F_{r}(q)}=\frac{\sum_{i=1}^{1000} F_{r}^{i}(q)}{1000}
$$

where the sufficient $i$ means $i$-th molecule generated in a computer.

We can choose a molecule among those generated by electronic computer, whose $F_{\mathrm{m}}(p)$ and $F_{\mathrm{r}}(q)$ are very similar to $\overline{F_{\mathrm{m}}(p)}$ and $\overline{F_{\mathrm{r}}(q)}$ theoretically calculated by eq 1 and 2 . Circles, squares and triangles in Figure 1 are the plots for such molecules. Then, we can conclude that these molecules $(i=839$ for $\mathrm{R}$ PAN, $i=59$ for $\gamma$-PAN-1 and $i=259$ for $\gamma$ -PAN-2) have the most probable meso- and racemo sequence distributions.

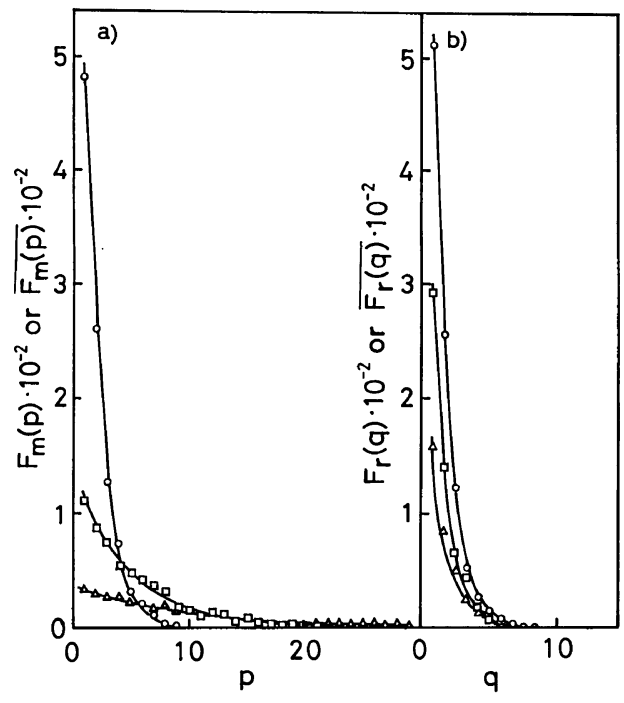

Figure 1. Dependence of $F_{\mathrm{m}}(p)$ or $F_{\mathrm{r}}(q)$ on $p$ or $q$ of RPAN and $\gamma$-PANs: full lines, $\overline{F_{\mathrm{m}}(p)}$ and $\overline{F_{\mathrm{r}}(q)}$ calculated using eq 1 and 2; a) $F_{\mathrm{m}}(p)$ generated using Monte Carlo method; b) $F_{\mathrm{r}}(q)$ generated using Monte Carlo method: O, R-PAN; $\square, \gamma$-PAN-1; $\triangle, \gamma$-PAN-2. 


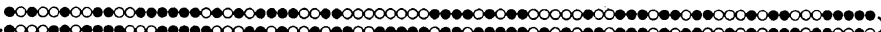

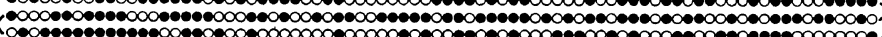

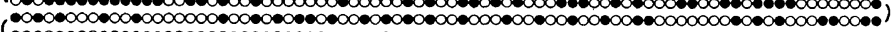
$\left(\begin{array}{ccc}* & 0\end{array}\right)$

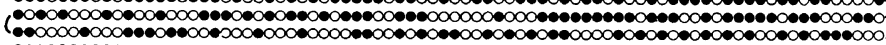

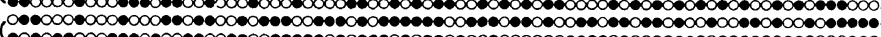

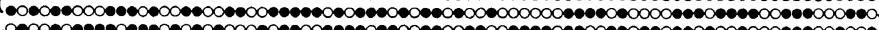

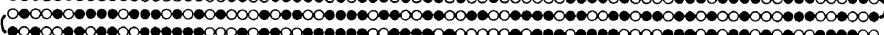

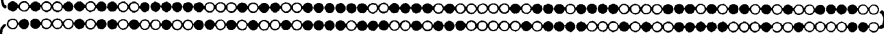

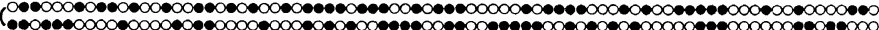

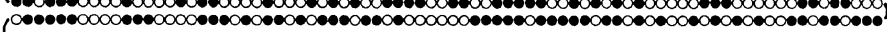

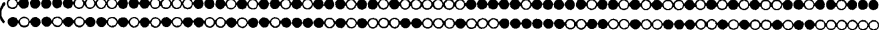

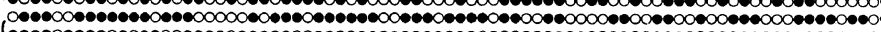
(

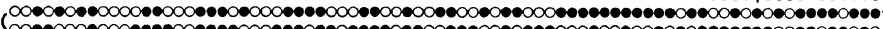

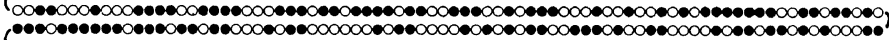

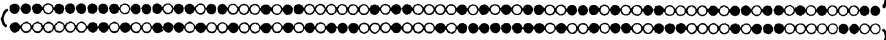

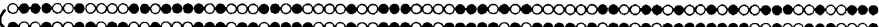
(1)

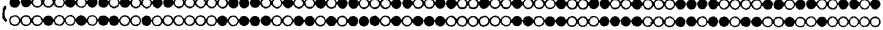

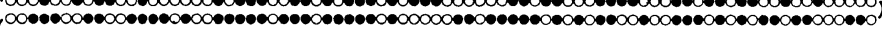
(OOO)

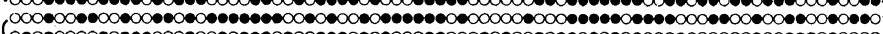

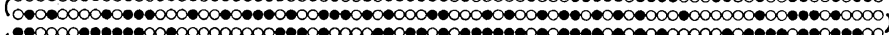

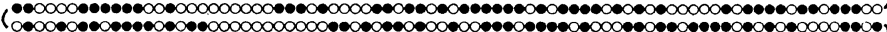

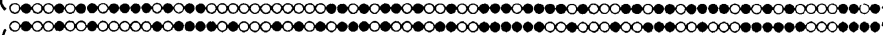

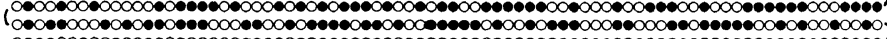

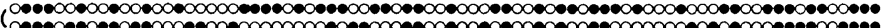
(1) ( (000000100

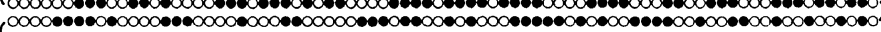

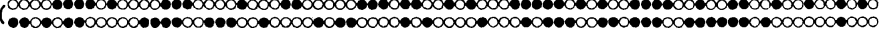

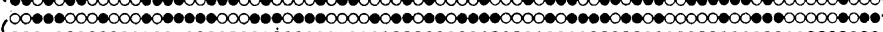

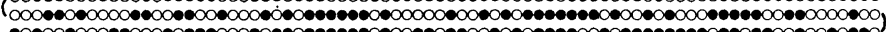

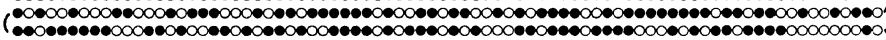

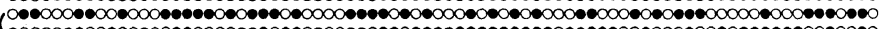

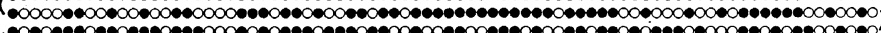

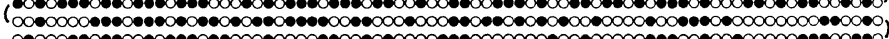
(

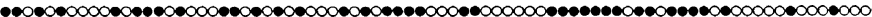

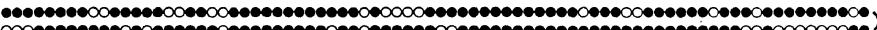
$($ (O)

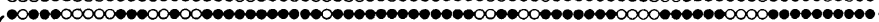
(

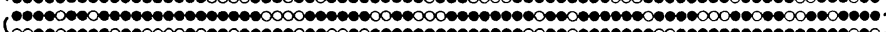

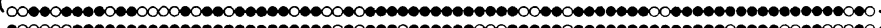
$(60000$

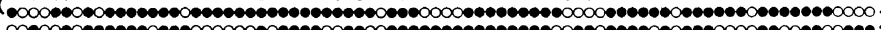

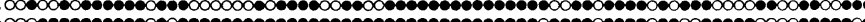
(1) (

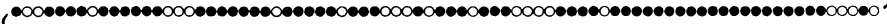
(

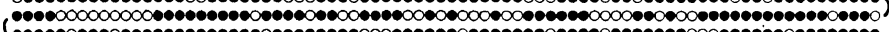
(

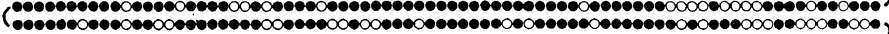

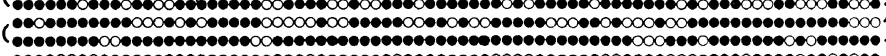

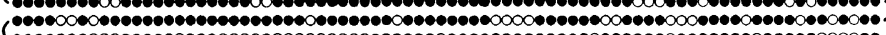

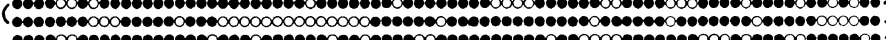
(

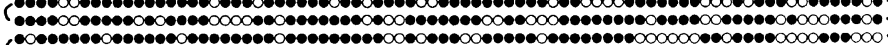
$(\bullet$ ( ( $(\bullet$ (

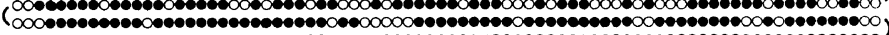
(

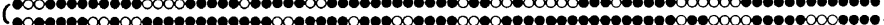
( (

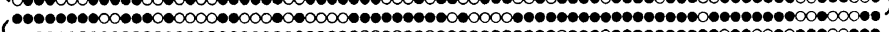
(

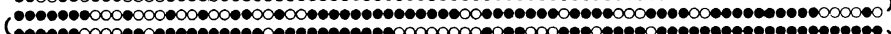
(

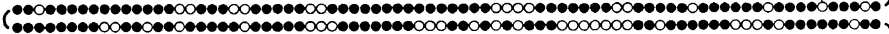

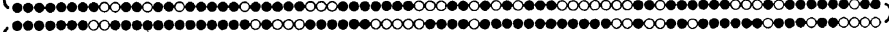

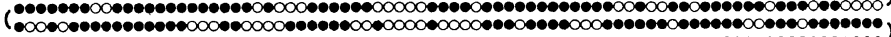

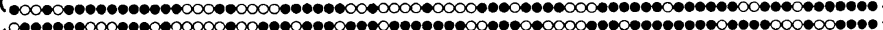

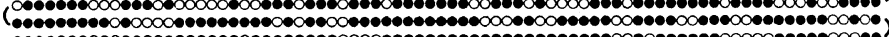
(

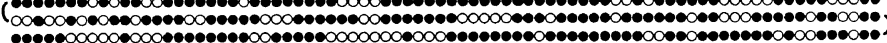




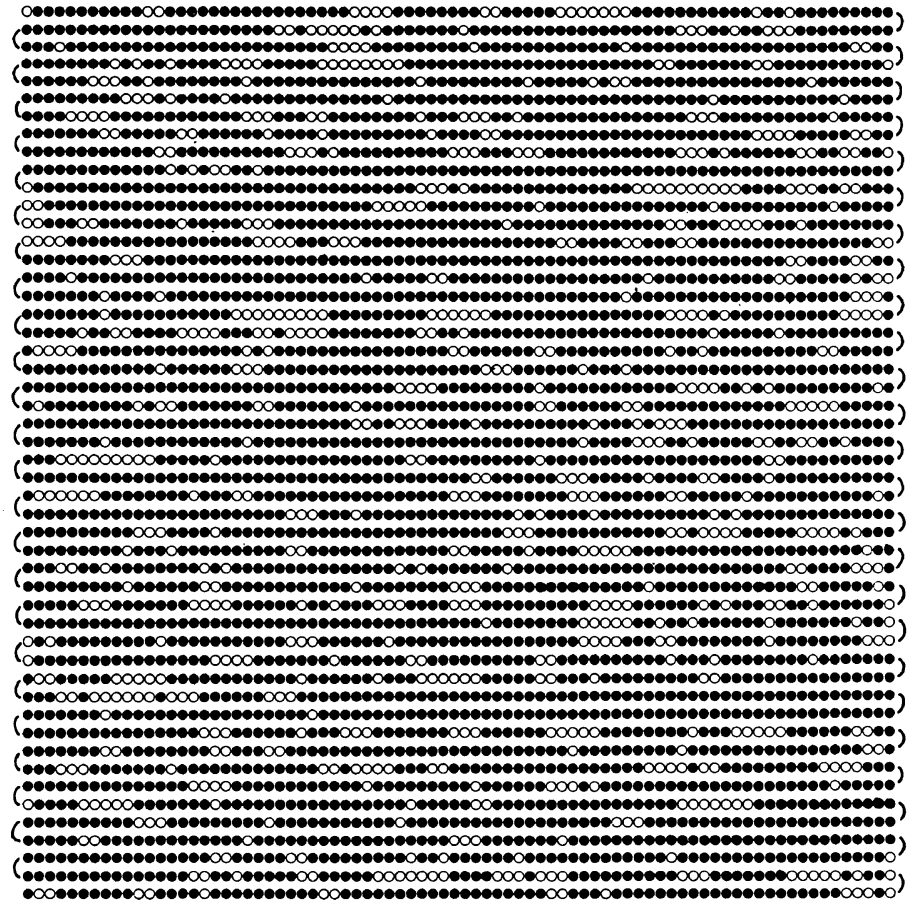

c

Figure 2. The most probable meso- and racemo-sequences of PANs, $N=4001$ : $\bigcirc$, meso dyad; $\bigcirc$, racemo dyad; a, R-PAN; b, $\gamma$-PAN-1; c, $\gamma$-PAN-2.

Here, the most probable polymer chain are selected in the manner that a parameter $\delta$ defined by

$$
\begin{aligned}
\delta= & \sqrt{\left.\sum_{p=1}^{N-1}\left(\overline{F_{m}(p)}\right)-F_{m}(p)\right)^{2}} \\
& +\sqrt{\left.\sum_{q=1}^{N-1}\left(\overline{F_{r}(q)}\right)-F_{r}(q)\right)^{2}}
\end{aligned}
$$

attains minimum.

Both $F_{\mathrm{m}}(p)$ of R-PAN and $F_{\mathrm{r}}(q)$ of R-PAN and $\gamma$-PAN decrease with increasing $p$ or $q$ very dramatically in the smaller $p$ and $q$ regions (i.e., $p, q<5) . \quad F_{\mathrm{m}}(p)$ of $\gamma$-PANs decreases with increasing $p$ gradually. $\gamma$-PAN has a possibility to have by far longer meso sequences than R-PAN, but the distribution of the racemo sequences of the former is not remarkably different from that of the latter.

Figure 2a, b, and c visualize the most probable meso and racemo sequences con- sisting a chain molecule of sample codes $\mathrm{R}$ PAN-1, $\gamma$-PAN-1, and $\gamma$-PAN-2 respectively, all generated in an electronic computer. In the figures, filled and unfilled circles mean meso and racemo sequences, respectively. An over-simplified illustration for $\gamma$-PAN-1 sample with the degree of polymerization of 104 was already given in a previous paper (see, Figure 3 of ref 2). Then, Figure 2 can be considered as highly sophisticated demonstration.

Very recently, the effect of stereoregularity on the dilute solution properties of PAN has been extensively investigated by Kamide et $a l{ }^{3}$ They concluded that unexpectedly larger conformation parameter $\sigma$ of PAN, which is some $35 \%$ larger than the expected value when simple steric hinderance due to the side chain group is considered, could be explained by considering the rigidity of the chain due to intra -interactions between neighbouring 
cyano-groups in meso sequence and the meso sequence distribution of PAN polymers, clarified in this study. In summarizing, the existence of relatively larger meso block in $\gamma$-PAN is a typical configuration characteristics and to a first approximation $\gamma$-PAN can be considered as a block copolymer of meso and racemo sequences.

\section{REFERENCES}

1. K. Kamide, H. Yamazaki, K. Okajima, and K. Hikichi, Polym. J., 17, 1233 (1985).

2. K. Kamide, H. Yamazaki, K. Okajima, and K. Hikichi, Polym. J., 17, 1291 (1985).

3. K. Kamide, H. Yamazaki, and Y. Miyazaki, Polym. J., 18, 819 (1986).

4. H. Yamazaki and K. Kamide, unpublished results. 